\title{
Keeping time
}

How finely can time be measured? The answer might define our capacity to conduct precise tests of general relativity, as demonstrated already in the observed increase in clock rate with distance from the Earth's surface, or the measurement of relativistic effects in binary pulsar systems. Spacecraft navigation, interferometric radio astronomy and Global Positioning System geodesy are all dependent on timekeeping that taxes current technology. And accurate measurements of the second define other standards, such as the units of distance and voltage.

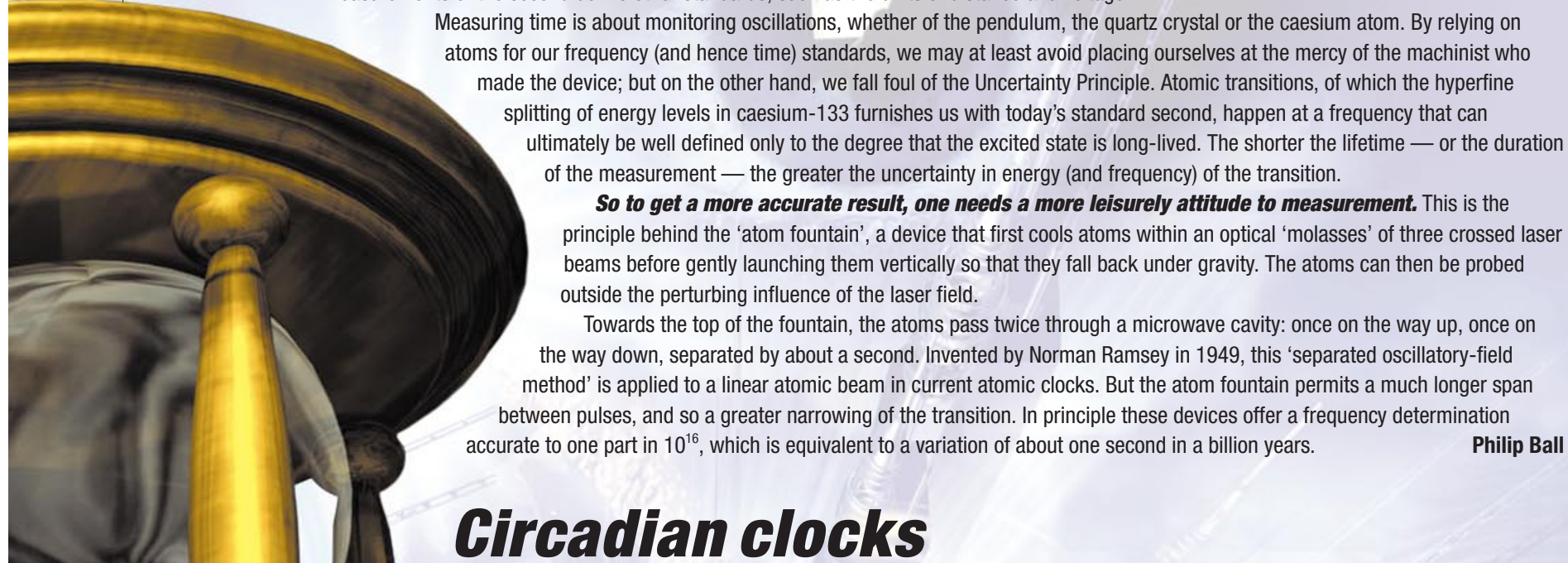

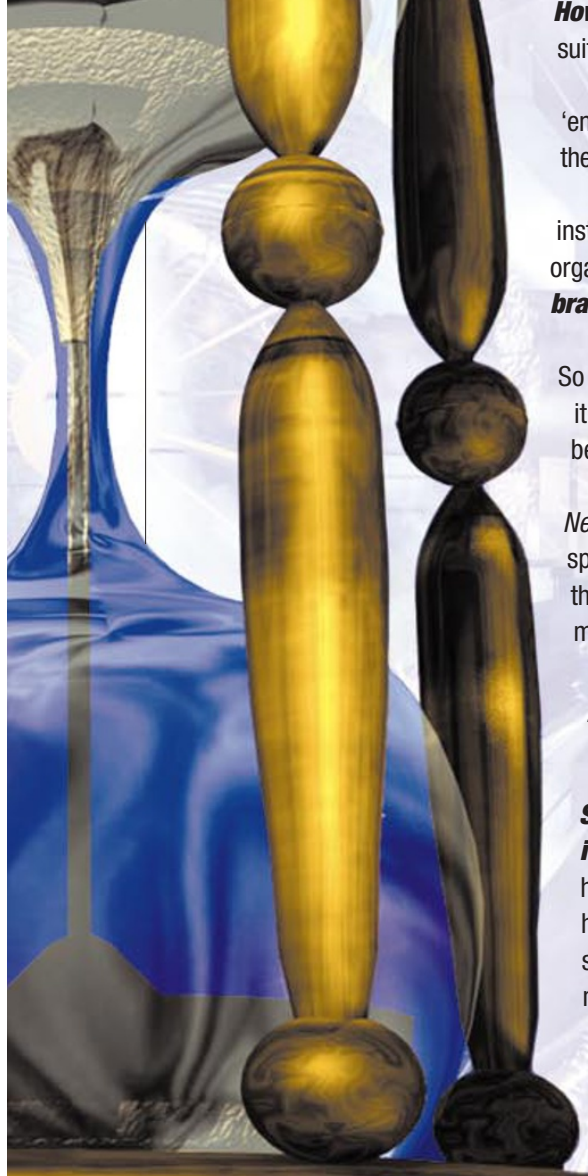

How do organisms respond to their most fundamental timescale - the Earth's rotation around its own axis? With a suite of interconnected hormonal and physiological loops that make up the 'circadian timing system' or CTS.

Every CTS - whatever the organism - is made up of light receptors, an endogenous 'biological' clock and a so-called 'entrainment' system to couple one with the other. For although every cell of every biological or circadian clock behaves cyclically, these intrinsic periods are not necessarily exactly 24 hours long. The clock has to be set.

Much of the pathology and endocrinology of the circadian clock has been elucidated in the past century. In humans, for instance, many sections of the route from retina to brain to heart-muscle action, metabolism, body temperature and the organization of sleep-wake cycles have been charted. But there are still major areas of uncertainty, especially in the newest branch of circadian biology: the identification and study of genes necessary for normal timekeeping.

Clock genes seem to be present in all cells, even though only a subset is capable of acting as self-sustained oscillators. So do these genes have other, global, non-circadian functions in cell biology? As Russell Foster of Imperial College, London, puts it: "cellular assays have shown the way forward, now we need good functional analysis using transgenic approaches and behavioural studies".

Moreover, only a few animal systems have been studied so far in any detail — the mouse, the fly and the fungus, Neurospora. This has revealed some remarkable similarities but also some exciting differences. Comparisons of additional species will surely tell us more about the evolution and adaptive significance of circadian systems and their interactions with their environments. All of which will hopefully feed back into a fine-tuned understanding of, and ability to treat or even to manipulate, the one timepiece that we all care about — the human body.

Sara Abdulla

\section{The challenge of conservation}

Some disciplines move faster than others, but if there is one moving much faster than its practitioners would like, it is conservation biology. In the next century, conservationists will be less interested in pristine wilderness - for such will hardly exist - but in the success with which human beings manage the biosphere to the benefit of all its inhabitants, including humans. The world is currently in an unprecedented situation in which Homo sapiens - just one species out of millions sequesters 45 per cent of the planet's net terrestrial productivity and 55 per cent of its fresh water. Effective biodiversity systems management will become an imperative if only to maintain the quality of human life, let alone that of other species.

In the coming decades, conservation biology will become a close analysis of the art of compromise, as humanity seeks

to understand the global ecosystem as a participant, not as an observer. "Within a century," says Peter Raven, director of the

Missouri Botanic Garden, "the world will be a patchwork with varying degrees of biological richness, and the way people respond locally to challenges will determine the nature of that compromise."

What will we have lost by 2100 ? Raven estimates that two-thirds of all species will disappear if current trends continue, but is nevertheless optimistic. "We can do better," he says. "No major category of

organisms, or local community of organisms, need disappear completely."

Ecologists are acutely aware that the maintenance of ecosystem function transcends the conservation of any particular species, so high-technology initiatives such as 'ex situ' conservation — maintaining banks of germplasm outside the context of a habitat — may become a side issue. "High technology is unlikely to solve most of the major global environmental issues that we face," says David Tilman of the University of Minnesota. "Rather, lifestyle changes, most likely imposed by legislation, will be needed. Such legislation will be socially sustainable only once citizens understand their long-term dependence on biodiversity and ecosystem services." And Raven adds that it is "better for now to follow Aldo Leopold's dictum: 'the first rule of intelligent tinkering is to save all the cogs and wheels'".

Henry Gee 